\title{
The utility of elastography and CEUS for the differentiation between benign and malignant cervical lymphadenopaty. Three cases report.
}

\author{
Daniela Fodor ${ }^{1}$, Isabela Pascu ${ }^{1}$, Sever Pop ${ }^{2}$, Laura Poanta ${ }^{1}$ \\ ${ }^{1} 2^{\text {nd }}$ Internal Medicine Department, ${ }^{2}$ ENT Department, "Iuliu Hatieganu” University of Medicine and Pharmacy, \\ Cluj-Napoca, Romania
}

\begin{abstract}
The differentiation between benign and malignant cervical lymph nodes is a frequent challenge for the clinician. We present the cases of three patients with cervical lymphadenopaty (inflammatory lymph node, lymphoma, and metastasis of laryngeal carcinoma) in which the use of elastography and contrast enhanced ultrasonography had substantial contribution to the correct final diagnosis.
\end{abstract}

Keywords: cervical lymph nodes, elastography, contrast enhanced ultrasonography

\section{Introduction}

Elastography is regarded as a promising tool for cervical lymph nodes examination, the lymph nodes in this location being easily accessible and can be efficiently compressed during ultrasound examination [1]. Contrast enhanced ultrasonography (CEUS) is now largely used in daily practice. For lymph node, CEUS evaluation was considered to give the possibility of discriminating between benign and malignant superficial lymph node only in special clinical settings [2]. The main problem of CEUS- the need for perfusion quantification and the use of these perfusion parameters as objective indicators for a specific disease- was largely studied. The development of specific software for quantification (VueBox, Bracco Suisse SA Switzerland or Qontrast, Esaote S.p.a, Florence, Italy) allows us now to have a better perspective for an objective evaluation by using CEUS [3]

Received 16.01.2013 Accepted 25.01.2013

Med Ultrason

2013, Vol. 15, No 1, 63-66

Corresponding author: Laura Poanta

Nicolae Pascaly $9 / 16$ str

400431, Cluj Napoca, Romania

Fax 0040264 596912, Phone 0040744894190,

Email: laurapoanta@yahoo.com
Our aim is to report three different clinical situations in which CEUS examination and perfusion quantification using Qontrast software (Esaote S.p.a, Florence, Italy) together with elastography had provided important data for a correct characterization of the cervical lymphadenopaty. All the cases were examined with GE Logiq S8 system, using grey scale ultrasonography (US), power Doppler, and elastography. For CEUS a bolus of $2.4 \mathrm{ml}$ SonoVue was administrated and video clips were recorded in the first 3 minutes after contrast agent was injected.

\section{Cases report}

\section{Case 1}

A 37 years old man, with previous rhinopharingeal cancer, presented with cervical palpable lymph node on the IIIrd level (middle jugular group). US showed an oval lymph node, shape index (mean short axis/mean long axis) $<0.5$, with hypoechoic asymmetrical thickened cortex, well-defined margins, and hilar vascular pattern. Elastographic evaluation found only small blue areas (hard areas) in less than $50 \%$ of the lymph node (fig 1a). CEUS examination revealed moderate homogenous enhancement. Quantitative analyse of contrast enhancement in the region of interest established a low peak of 

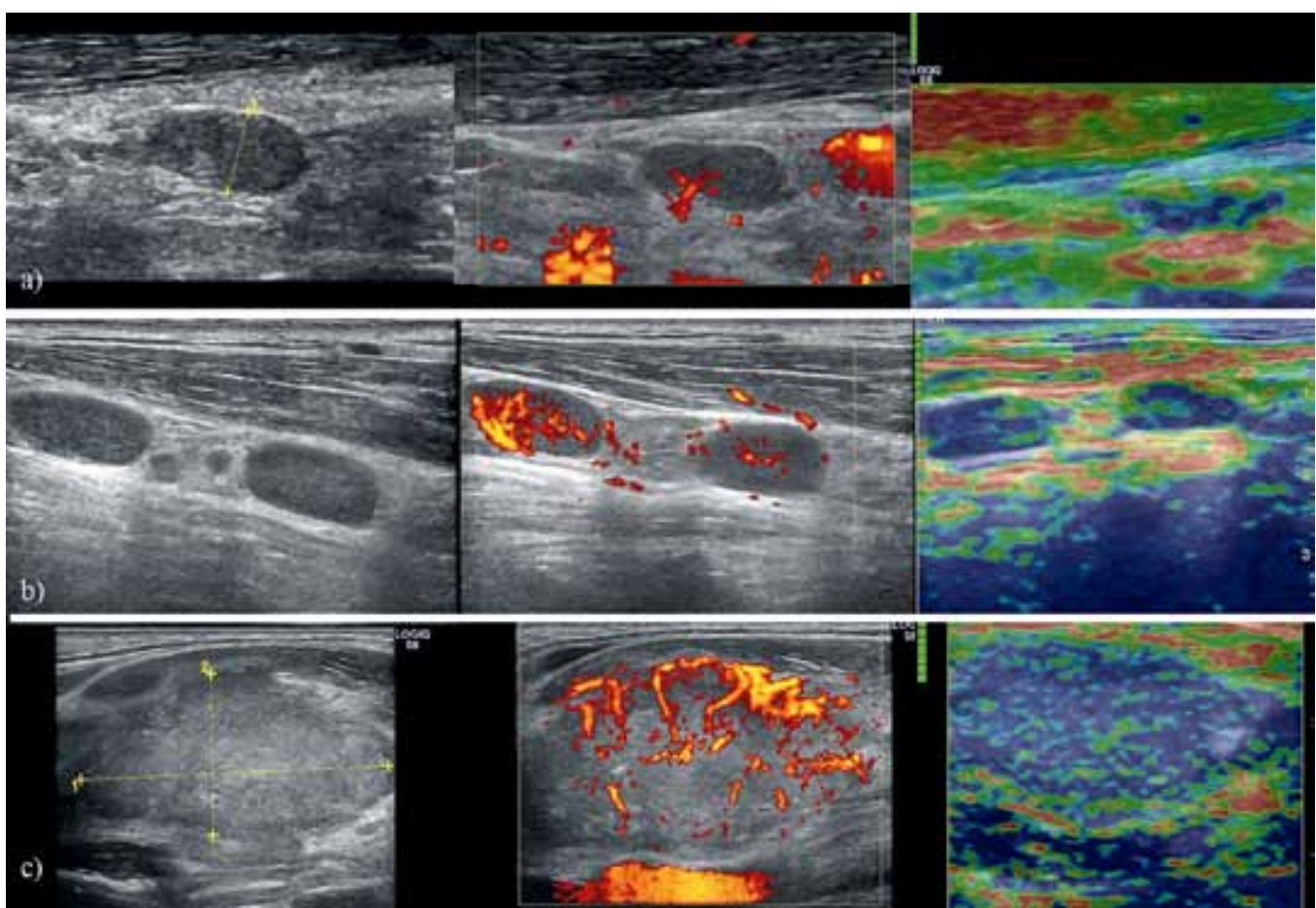

Fig 1. US aspect (grey scale, power Doppler, and elastography) of the three cases: a) case 1- inflammatory lymph node; b) case 2- T cell lymphoma; c) cases 3- metastasis from laryngeal cancer (explications into text)
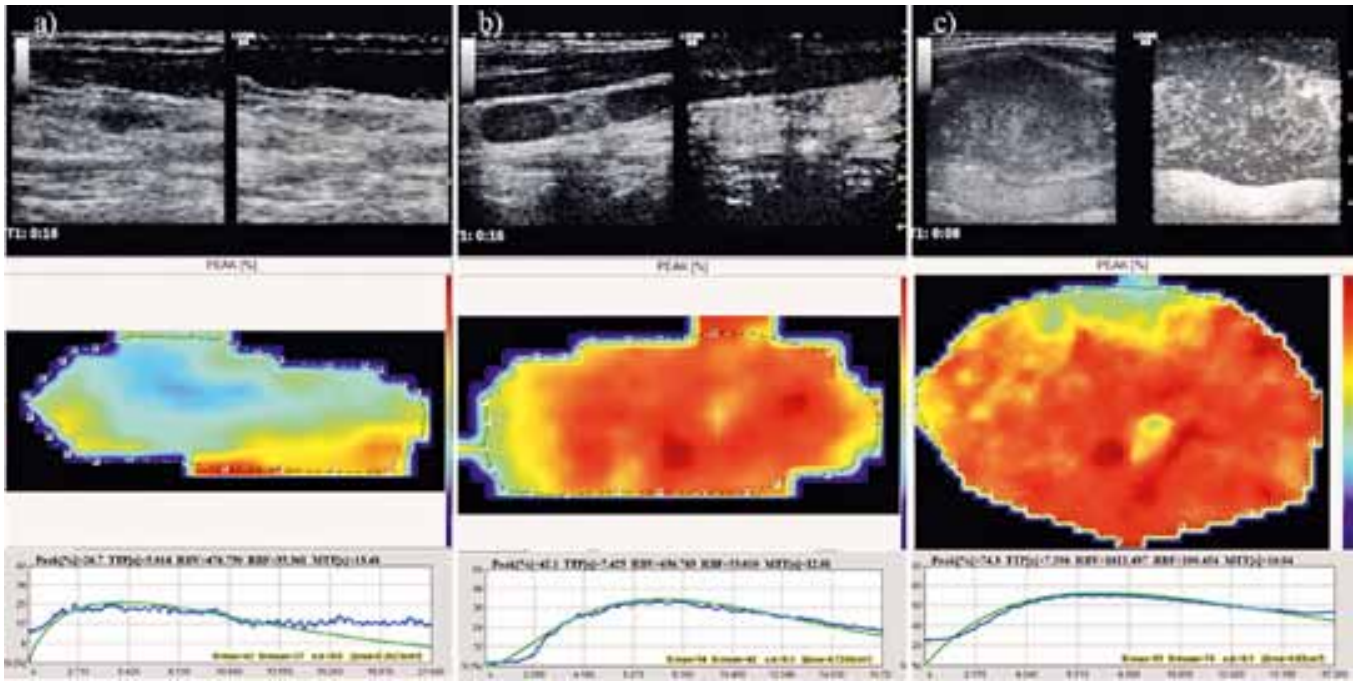

Fig 2. CEUS examination, peak of signal intensity map, and CEUS parameters of the three cases: a) case 1- inflammatory lymph node; b) case 2- T cell lymphoma; c) cases 3- metastasis from laryngeal cancer (explications into text) 
the signal intensity $(26.7 \%)$ and regional blood volume of 476.7 (fig 2a). Histopathological examination after surgical removement described the aspect of reactive, non-specific lymph node.

\section{Case 2}

A 59 years old man presented with multiple left cervical lymphadenopaty (between $1-1.5 \mathrm{~cm}$ ) in the upper and middle jugular groups. US examination determined the presence of multiple oval (shape index $<0.5$ ), hypoechoic, homogenous lymph nodes, no demonstrable hilum, and hilar pattern of vascularization. Elastography identified large blue areas (stiff areas), in more than $50 \%$ of the lymph node, especially in the periphery (fig $1 \mathrm{~b}$ ). Contrast agent administration produced an intense homogenous enhancement with central pattern, the peak of the signal intensity of $42.1 \%$, and regional blood volume of 636.8 (fig 2b). Surgical histology showed T cell lymphoma.

\section{Case 3.}

A 51 years old man was admitted with a palpable lymph node in the middle jugular group. A large, slightly hyperechoic and moderate heterogeneous lymph node was found on US examination. The margins were ill-defined, shape index $>0.5$, and the vascularization pattern was peripheral and disorganized. Hard areas (blue areas) occupied almost entire lymph node at elastography (fig 1c). CEUS showed an intense inhomogeneous enhancement with peripheral pattern, high peak of the signal intensity of $74.3 \%$, and high regional blood volume of 1611.6 (fig 2c). Histopathologic examination of the US guided biopsy sample determined the aspect of metastasis from a laryngeal carcinoma.

\section{Discussions}

Lymph node of the head and cervical region are always a challenge for the clinician, especially in cases in which the differential diagnostic between benign and malignant pathology is imposed. Ultrasonography is the first imaging technique in these cases due to a good accessibility of the cervical region, high spatial resolution, and a soft tissue discrimination $[4,5]$.

The criteria for gray scale and color/power Doppler US were developed for differentiation between different types of lymph nodes. The oval shape (shape index $<0.5$ or Solbiati index- ratio of the longitudinal to the short axis diameters- above than 2 or 1.5), the homogenous structure, the uniform cortical width, the hyperechoic hilum, the well defined margins, and the hilar vascular pattern are characteristics of inflammatory lymph nodes. Signs of malignant lymph nodes are considered to be the hypoechoic and heterogeneous texture, the round shape, the lack of hyperechoic hilum, the ill-defined margins, and the peripheral or mixed pattern of vascularization $[4,5]$. Until now no absolute criterion has been defined due to the false positive and false negative aspects encountered in normal and pathological lymph nodes $[5,6]$.

In the last years the development of elastography and CEUS added supplementary data for the lymph node characterization [7]. Analysis of elastography data combined with B-mode sonography proved to have $92 \%$ sensitivity, 94\% specificity, and 93\% accuracy in a correct interpretation of the cervical lymph nodes [8] with good agreement between observers [9]. Hard area in the lymph node in more than $45 \%$ [8] or $50 \%$ [10] is considered to be criteria for malignancy. Intense homogenous enhancement pattern is described for benign lymph nodes or lymphoma and inhomogeneous enhancement for metastases [11]. The different types of lymphoma seems to have a more variable aspect: central type pattern of vascularization or lack of enhancement in B cell lymphoma and peripheral type pattern of vascularization or intense homogenous enhancement in T cell lymphoma $[11,12]$.

In our case 1, the personal history of the patient and the asymmetrical thickened cortex on grey-scale US raised the susceptibility of malignant lymph node. The elastographic aspect of soft lymph node and CEUS with moderate and homogenous enhancement allowed us to interpret it as a benign structure, aspect confirmed by histopathology. The low peak of signal intensity and the regional blood volume had contributed to this interpretation. In case 2 the oval shape, with shape index $<0.5$, homogeneity and hilar pattern of vascularization were characteristics of benign lymph node. The absence of the echogenic hilum was not considered a sign of malignancy, knowing that the normal lymph node don't often have hyperechoic hilum in head and neck region ${ }^{4}$. The hard (stiff) aspect on elastography and intense homogenous enhancement with high peak intensity were considered signs of malignancy. In the third case the aspect of malignant lymph node was obvious on grey-scale and power Doppler US examination. The hard aspect of the entire lymph node on elastography, the intense inhomogeneous enhancement with peripheral pattern, and the very high parameters on quantitative CEUS analysis suggested the aspect of metastasis and not lymphoma. These cases show the importance of a complete US examination of the lymph nodes, using all the US techniques.

In conclusion elastography and CEUS seem to be promising tools for the benign-malignant lymph nodes differentiation and more studies on quantification of CEUS parameters could bring important quantitative criteria in diagnosis. 
Sources of funding: This work was carried out with funds from "Iuliu Hatieganu" University of Medicine and Pharmacy, Cluj-Napoca, Romania 27020/47/15.11.2011 grant

\section{References}

1. Das D, Gupta M, Kaur H, Kalucha A. Elastography: the next step. J Oral Sci 2011;53:137-141.

2. Piscaglia F, Nolsøe C, Dietrich CF, et al. The EFSUMB Guidelines and Recommendations on the Clinical Practice of Contrast Enhanced Ultrasound (CEUS): update 2011 on non-hepatic applications. Ultraschall Med 2012;33:33-59.

3. Tranquart F, Mercier L, Frinking P, Gaud E, Arditi M. Perfusion quantification in contrast-enhanced ultrasound (CEUS)-ready for research projects and routine clinical use. Ultraschall Med 2012;33 Suppl 1:S31-38.

4. Gupta A, Rahman K, Shahid M, et al. Sonographic assessment of cervical lymphadenopathy: role of high-resolution and color Doppler imaging. Head Neck 2011;33:297-302.

5. Rettenbacher T. Sonography of peripheral lymph nodes part 1: normal findings and B-image criteria. Ultraschall Med 2010;31:344-362.

6. Catalano O. Critical analysis of the ultrasonographic criteria for diagnosing lymph node metastasis in patients with cutaneous melanoma: a systematic review. J Ultrasound Med 2011;30:547-560.

7. Dudea SM, Lenghel M, Botar-Jid C, Vasilescu D, Duma M. Ultrasonography of superficial lymph nodes: benign vs. malignant. Med Ultrason 2012;4:294-306

8. Alam F, Naito K, Horiguchi J, Fukuda H, Tachikake T, Ito $\mathrm{K}$. Accuracy of sonographic elastography in the differential diagnosis of enlarged cervical lymph nodes: comparison with conventional B-mode sonography. AJR Am J Roentgenol 2008;191:604-610.

9. Lenghel LM, Bolboacă SD, Botar-Jid C, Baciut G, Dudea $\mathrm{SM}$. The value of a new score for sonoelastographic differentiation between benign and malignant cervical lymph nodes. Med Ultrason 2012;4:271-277.

10. Taylor K, O'Keeffe S, Britton PD, et al. Ultrasound elastography as an adjuvant to conventional ultrasound in the preoperative assessment of axillary lymph nodes in suspected breast cancer: a pilot study. Clin Radiol 2011;66:10641071.

11. Yu M, Liu Q, Song HP, et al. Clinical application of contrast-enhanced ultrasonography in diagnosis of superficial lymphadenopathy. J Ultrasound Med 2010;29:735-740.

12. Nakase K, Yamamoto K, Hiasa A, Tawara I, Yamaguchi $\mathrm{M}$, Shiku H. Contrast-enhanced ultrasound examination of lymph nodes in different types of lymphoma. Cancer Detect Prev 2006;30:188-191. 